\title{
Medizin-ethische Richtlinien zur Feststellung des Todes
}

\section{Michelle Salathé}

lic. iur. MAE, stv. Generalsekretärin SAMW

\author{
Die sichere Feststellung des Todes ist eine zentrale Voraussetzung für die Transplan-
} tationsmedizin, soweit diese auf der Organentnahme bei Verstorbenen beruht. Sie hat enorme ethische, rechtliche und politische Bedeutung, denn sie muss garantieren, dass Lebende immer als Lebende und Tote immer als Tote behandelt werden. Die SAMW-Richtlinien Feststellung des Todes mit Bezug auf Organtransplantationen (Mai 2011) werden revidiert und sind bis Ende Februar 2017 in Vernehmlassung.

Zur sicheren Feststellung des Todes ist ein klares und allgemeingültiges Kriterium erforderlich, das den Eintritt des Todes definiert. Zugleich braucht es eine zuverlässige Methode, um den Tod anhand dieses Kriteriums festzustellen. Das Transplantationsgesetz (TxG) legt als Kriterium für den Tod eines Menschen den irreversiblen Ausfall der Funktionen seines Hirns einschliesslich des Hirnstamms fest. Für das klinische Vorgehen zur Feststellung des Todes verweist die Verordnung zum Transplantationsgesetz auf die entsprechenden Abschnitte der Richtlinien Feststellung des Todes mit Bezug aufOrgantransplantationen der Schweizerischen Akademie der Medizinischen Wissenschaften (SAMW) vom 24. Mai 2011.

Im Juni 2015 verabschiedete das Parlament eine revidierte Fassung des TxG. Diese tritt in Kraft, sobald die notwendigen Anpassungen im Verordnungsrecht erfolgt sind. Am 21. Oktober 2016 hat das EDI die entsprechenden Entwürfe in die Vernehmlassung gesandt, die noch bis zum 3. Februar 2017 dauert. Die Revision des TxG erfordert eine Überarbeitung der SAMW-Richtlinien. Deshalb hat die Zentrale Ethikkommission der SAMW (ZEK) eine Subkommission ${ }^{1}$ unter dem Vorsitz von Prof. Jürg Steiger, Basel, damit beauftragt, die notwendigen Anpassungen vorzunehmen.

Anlass für die Revision des TxG war eine Motion zur Zuteilung der gespendeten Organe an Grenzgängerinnen und -gänger. Im Verlauf der Revision wurden wichtige Anliegen aus der Praxis aufgenommen, darunter die Frage, ab wann Angehörige einbezogen werden bzw. ob Angehörige bereits vorbereitenden medizinischen Massnahmen vor dem Tod zustimmen können oder nicht. Die revidierten Richtlinien gehen auf diese zentralen Punkte im Detail ein und bieten neu u.a. eine Visualisierung (Flowchart) des Entscheidungspro- zesses (Anhang B). Eine Übersicht über die zentralen Revisionspunkte bieten die Fragen und Antworten auf der nachfolgenden Seite.

Bereits in der Ausarbeitungsphase hat die Subkommission Expertinnen und Experten, namentlich Vertreterinnen und Vertreter der Entnahmespitäler und Organspendenetzwerke, der betroffenen Fachgesellschaften und der Pflege, aber auch die Patientenorganisationen konsultiert und deren Rückmeldungen berücksichtigt. Der Senat hat den Richtlinienentwurf am 16. November 2016 zur Vernehmlassung verabschiedet. Die Vernehmlassung ist bis Ende Februar 2017 offen, interessierte Organisationen und Personen sind zur Stellungnahme eingeladen.

Unverändert bleiben die folgenden zentralen Punkte der Richtlinien:

- Voraussetzungen des Todeskriteriums (klinische Zeichen);

- Untersuchung durch zwei Fachärzte (Vier-AugenPrinzip), kein Zeitintervall;

- Eine technische Zusatzuntersuchung ist nur erforderlich, wenn die Voraussetzungen für die klinische Untersuchung nicht erfüllt sind (d.h. wenn die Hirnnerven klinisch nicht untersucht oder potentiell reversible Ursachen als Mitursachen nicht ausgeschlossen werden können);

- Keine Organentnahme bei Neugeborenen.

Vernehmlassungsentwurf und weitere Informationen

www.samw.ch $\rightarrow$ Ethik $\rightarrow$ Transplantation $\rightarrow$ Überarbeitung der Richtlinien 


\section{Die zentralen Revisionspunkte der Richtlinien zur Feststellung des Todes}

\section{Wann dürfen die Angehörigen zur Organspende befragt werden?}

Die klare Trennung zwischen der Entscheidung, eine lebenserhaltende Therapie abzubrechen (Änderung des Behandlungsziels), einerseits und der Entscheidung, Organe $\mathrm{zu}$ spenden, muss sichergestellt sein. Auch wenn Angehörige das Thema der Organspende frühzeitig ansprechen, darf die Zustimmung zur Organentnahme und zu allfälligen vorbereitenden medizinischen Massnahmen erst eingeholt werden, nachdem der Entscheid feststeht, die lebenserhaltenden Therapien abzubrechen. Die Gespräche dürfen aber bereits vor der Feststellung des Todes geführt werden.

Welche Voraussetzungen müssen erfüllt sein, bevor eine Feststellung des Todes durchgeführt werden kann? Aufgrund von Rückmeldungen aus der Praxis werden in einem Anhang die klinischen Voraussetzungen umschrieben, die erfüllt sein müssen, bevor die Feststellung des Todes durchgeführt werden darf (Anhang C).

\section{Wer darf den Tod feststellen?}

Die klinische Beurteilung muss durch Fachärzte mit Weiterbildung und einer nachweisbar ausreichenden Erfahrung erfolgen. Eine ausreichende Erfahrung ist dann gegeben, wenn mindestens einer der beiden die
Diagnostik durchführenden Fachärzte bei mindestens fünf Patienten eine Hirntoddiagnostik unter Supervision durchgeführt hat.

\section{Welche vorbereitenden Massnahmen dürfen vor dem Tod durchgeführt werden?}

Gemäss revidiertem TxG dürfen vorbereitende Massnahmen vor dem Tod des Spenders durchgeführt werden, wenn dieser selbst eingewilligt hat oder die Angehörigen dazu einwilligen, sofern ihnen keine Ablehnung des Spenders bekannt ist. Neu wird der komplizierte Entscheidungsprozess in einem Flowchart dargestellt (Anhang B). Die Richtlinien zählen zudem in einer Negativliste (Anhang $\mathrm{H}$ ) abschliessend diejenigen vorbereitenden Massnahmen auf, die in keiner Situation durchgeführt werden dürfen, weil sie für eine erfolgreiche Transplantation nicht unerlässlich und mit mehr als minimalen Risiken verbunden sind.

Was gilt, wenn Angehörige aus persönlicher Überzeugung eine Organentnahme ablehnen, obwohl eine Spendekarte vorliegt?

Die Richtlinien verweisen auf die rechtliche Regelung, die festhält, dass der Wille der verstorbenen Person demjenigen der nächsten Angehörigen vorgeht. Sie empfiehlt aber, wenn immer möglich eine einvernehmliche Entscheidung unter allen Beteiligten anzustreben und notfalls eine ethische Unterstützung beizuziehen. 